\title{
EDITORIAL
}

\section{Mental Health Disorders: The Deserted Illnesses}

\section{Abraham Haileamlak, MD, Professor of Pediatrics and Child Health}

According to the World Health Organization's (WHO's) definition, "Mental health is a state of well-being in which an individual can realize his or her own abilities, interact positively with others, cope with the stressors of life and study, work productively and fruitfully, and contribute to his or her family and community." In short WHO affirms that "There is no health without mental health." However, because of familial predisposition, stressful life, substance use and other reasons, mental health disorders are increasing worldwide.

Mental health disorders have been a significant public health problem in low or middle income countries (LMICs) including Ethiopia. This being the case, these disorders have received disproportionate attention because of the high burden of communicable diseases and malnutrition. Besides the burden they impose on the health system, mental health disorders contribute to lost worker productivity, poor functioning, personal stigma and familial and social stress (1-2).

Although there are proven strategies that help reduce morbidity and disability associated with mental health disorders $(3,4)$, those who receive appropriate treatment remained low in many LMICs(5) where treatment options and access to treatment are suboptimal (6). The main reason for this is the limited number of mental healthcare professionals and limited access to modern medicines for such diseases. Report showed that many countries in Africa and the South-East Asia have fewer psychiatrists and psychiatry nurses (7).

Although there are numerous mental health disorders requiring attention, the WHO's Mental health Gap Action Program (mhGAP) in 2008 identified eight major problems (depression, schizophrenia, psychoses other than schizophrenia, suicide, epilepsy, dementia, alcohol and illegal drugs abuse, and pediatric mental disorders) that contribute to $75 \%$ of the global burden of mental disorders that LMICs should prioritize (8). The mental health disorders listed above have been common and are the major conditions which substantially undermine children's learning skills and adults' abilities to function within the family and the society (7).

For several years, mental health champions have been advocating that countries increase their mental health workforce. Similarly, WHO's call for a scale-up of mental health work force in the mhGAP report $(8,9)$. Unfortunately, progresses in producing health workforce for the care of mental illnesses and integration of the mental health care in existing health facilities have been slow (12). Still, it is not very late for healthcare planners to improve the human resources required to meet the mental healthcare needs of their populations. LMICs may need to device strategy that can heighten efficiency of the existing workforce like task-sharing, which is found to be effective in other healthcare services $(10,11)$.

In the last two decades, Ethiopia made several efforts to increase mental health professionals and to integrate mental health services in primary healthcare. Accordingly, a five year mental health strategy, the first of its kind, was developed and implemented (12). Yet, despite those efforts, both primary healthcare professionals who are capable of providing basic mental health care services and high level mental health professionals (mental health nurses and psychiatrists) are very few and unevenly distributed (13). Modern medicines being used in most developed world in treating such conditions are either totally unavailable or inconsistently available. Because of this, leave alone the wider mental health disorders, the WHO prioritized mental health problems remain unaddressed. While rapid rise in the numbers of health

DOI: http://dx.doi.org/10.4314/ejhs.v27i1.1 
professional training institutions and health care facilities was demonstrated in the last two decades, only two health professional training institutions are producing mental health care professionals which resulted in highly unmeet mental health treatment requirement.

The current issue (Vol. 27, No 1), the first regular issue for the year, contains one editorial, eight original articles, one meta-analysis and four case reports. The editorial and three of the original articles focus on the ever increasing condition-mental health disorders. The other articles and case reports cover various topics. I invite readers to go through these articles and appreciate or utilize the contents. I also encourage readers to forward comments and suggestions to the editor or corresponding authors.

\section{REFERENCES}

1. Hu TW. The economic burden of depression and reimbursement policy in the Asia Pacific region. Australas

Psychiatry2004;12(Suppl):S11-5.

2. Tsang HWH, Tam PKC, Chan F, Cheung WM. Sources of burdens on families of individuals with mental illness. Int J Rehabil Res 2003;26:123-30. doi:10.1097/00004356200306000-00007

3. WHO. Disease control priorities related to mental, neurological, developmental and substance abuse disorders.World Health Organization; Geneva: 2006.

4. Patel V, Araya R, Chatterjee S, et al. Treatment and prevention of mental disorders in low-income and middle-income countries. Lancet 2007;370:991-1005. doi:10.1016/S0140-6736(07)61240-9

5. Wang PS, Aguilar-Gaxiola S, Alonso J, et al. Use of mental health services for anxiety, mood, and substance disorders in 17 countries in the WHO world mental health surveys. Lancet 2007;370:841-50. doi:10.1016/S0140-6736(07)61414-7

6. Kohn R, Saxena S, Levav I, Saraceno B. The treatment gap in mental health care. Bull World Health Organ 2004;82:858-66.
7. WHO. The mental health workforce gap in low- and middle-income countries: a needsbased approach. Bull World Health Organ 2011;89 (3). Doi http://dx.doi.org/10.2471/BLT.10.082784

8. WHO.mhGAP: mental Health Action Programme: scaling up care for mental,neurological, and substance use disorders. World Health Organization; Geneva:2008.

9. WHO. The world health report 2001: mental health: new understanding, new hope. Geneva: World Health Organization; 2001.

10. Saraceno B, van Ommeren M, Batniji R, et al. Barriers to improvement of mental health services in low-income and middleincome countries. Lancet 2007;370:1164-74. doi:10.1016/S0140-6736(07)61263-X

11. Jacob KS, Sharan P, Mirza I, et al. Mental health systems in countries: where are we now? Lancet 2007;370:1061-77. doi:10.1016/S0140-6736(07)61241-0

12. Federal Democratic Republic of Ethiopia Ministry of Health. National Mental Health Strategy 2012/13 - 2015/16.

13. Abebaw Fekadu, Graham Thornicroft. Global Mental Health: PerspectivesFrom Ethiopia. Glob Health Action 2014;7. http://dx.doi.org/10.3402/gha.v7.254 47. 